\title{
Gold Catalysis for Selective Hydrogenation of Aldehydes and Valorization of Bio-Based Chemical Building Blocks
}

\author{
Rerison J. M. Silva, ${ }^{a}$ Jhonatan L. Fiorio, ${ }^{a}$ Pedro Vidinha ${ }^{a}$ and Liane M. Rossi ${ }^{\oplus * a}$ \\ ${ }^{a}$ Departamento de Química Fundamental, Instituto de Química, Universidade de São Paulo, \\ Av. Prof. Lineu Prestes, 748, 05508-000 São Paulo-SP, Brazil
}

\begin{abstract}
Gold catalysts are best known for their selectivity in oxidation reactions, however, there is a promising future for gold in selective hydrogenations. Herein, the hydrogenation of several aldehydes and important bio-based chemical building blocks, namely 5-hydroxymethylfurfural (5-HMF), furfural and vanillin, was performed throughout the combination of Au nanoparticles with Lewis bases. The Au-amine ligand (e.g., 2,4,6-trimethylpyridine) catalytic system could reduce the aldehyde carbonyl group selectively, without reducing alkene moieties or opening the furanic ring that occur on most traditional catalysts. Otherwise, the reduction of nitro group is preferential and the catalytic system was used for the synthesis of furfurylamines, important intermediates in the synthesis of different pharmaceuticals (e.g., furosemide), through the selective reductive amination of furfural starting from nitro-compounds. Moreover, a fully heterogeneous gold catalyst embedded in N-doped carbon ( $\mathrm{Au} @ \mathrm{~N}$-doped carbon / $\mathrm{TiO}_{2}$ ) was able to perform these reactions in successive recycles without the addition of ligands, with impact in the development of a continuous flow process for biomass valorization.
\end{abstract}

Keywords: gold, heterogeneous catalysis, N-doped carbon, hydrogenation, aldehydes

\section{Introduction}

Valorization of biomass platform molecules, such as 5-hydroxymethylfurfural (5-HMF) and furfural (FA), has been explored via selective oxidation, ${ }^{1}$ esterification, ${ }^{2}$ amination $^{3}$ and hydrogenation. ${ }^{4}$ The main drawback in a hydrogenative route is the hydrogenolysis and / or ring opening reaction that occur on most traditional catalysts (Scheme 1). ${ }^{5}$ Among other products, the selective hydrogenation of 5-HMF into 2,5-dihydroxymethylfuran (DHMF), the analog of $m, p$-benzenedimethanol, which is commonly used for the production of resins, polyesters and polyurethanes, is particularly interesting. ${ }^{2,4}$ However, this product is difficult to obtain in high yields. Bottari et al. ${ }^{6}$ investigated the catalytic hydrogenation of 5-HMF with copper nanocatalysts of various compositions and found that the catalyst mostly led to a mixture of products in which dimethylfuran (DMF) and 2,5-dimethyltetrahydrofuran (DMTHF) were the major products, as well as etherification and ring opening products followed by hydrogenation as by-products (ca. $20 \%$ yield). In order to improve conversion and selectivity, the authors chose the best performance catalyst $(\mathrm{CuZn})$ and varied reaction conditions

*e-mail: 1rossi@iq.usp.br (temperature, pressure, solvent, etc.) and finally managed to improve the conversion to around $90 \%$ and formation of only DMF and DMTHF under high temperature conditions $\left(220{ }^{\circ} \mathrm{C}\right)$ and reasonably high pressure $\left(20\right.$ bar $\left.\mathrm{H}_{2}\right)$. More recently, Chen et al. ${ }^{7}$ reported the use of $\mathrm{Pd}$ nanoparticles (NPs) supported on mesoporous graphitic carbon nitride $\left(\mathrm{Pd} / \mathrm{mpg}_{-} \mathrm{C}_{3} \mathrm{~N}_{4}\right)$ for the hydrogenation of 5-HMF leading to the formation of dihydromethyl tetrahydrofuran (DHMTF). In addition, the use of $\mathrm{Ru}$ NPs $\left(\mathrm{Ru} / \mathrm{mpg}-\mathrm{C}_{3} \mathrm{~N}_{4}\right)$ was also reported, which led to the formation of a mixture of DHMTF and hexanediol (HD) (ring opening products) with selectivities of 60 and $40 \%$, respectively.

It has been recently demonstrated that the dissociation of $\mathrm{H}_{2}$, which is limited on gold as compared to the platinum group metals, can be promoted by an activation mechanism via frustrated Lewis pairs (FLPs). ${ }^{8-11}$ The pioneering studies on the hydrogenation of carbonyl compounds by gold were not as efficient as the FLPs formed at the metal-ligand interface. ${ }^{12}$ After the discovery of this heterolytic pathway for activation of $\mathrm{H}_{2}$ on gold, the reduction of carbonyl groups of aldehydes, ketones, esters and amides, ${ }^{13-16}$ semihydrogenation of alkynes, ${ }^{17-19}$ nitro, ${ }^{20}$ quinolines, ${ }^{21}$ allylic carbonyls, ${ }^{22}$ epoxides were made easy. ${ }^{23}$

On most metals, the hydrogenation of $\mathrm{C}=\mathrm{C}$ double bonds is thermodynamically more favorable than the 


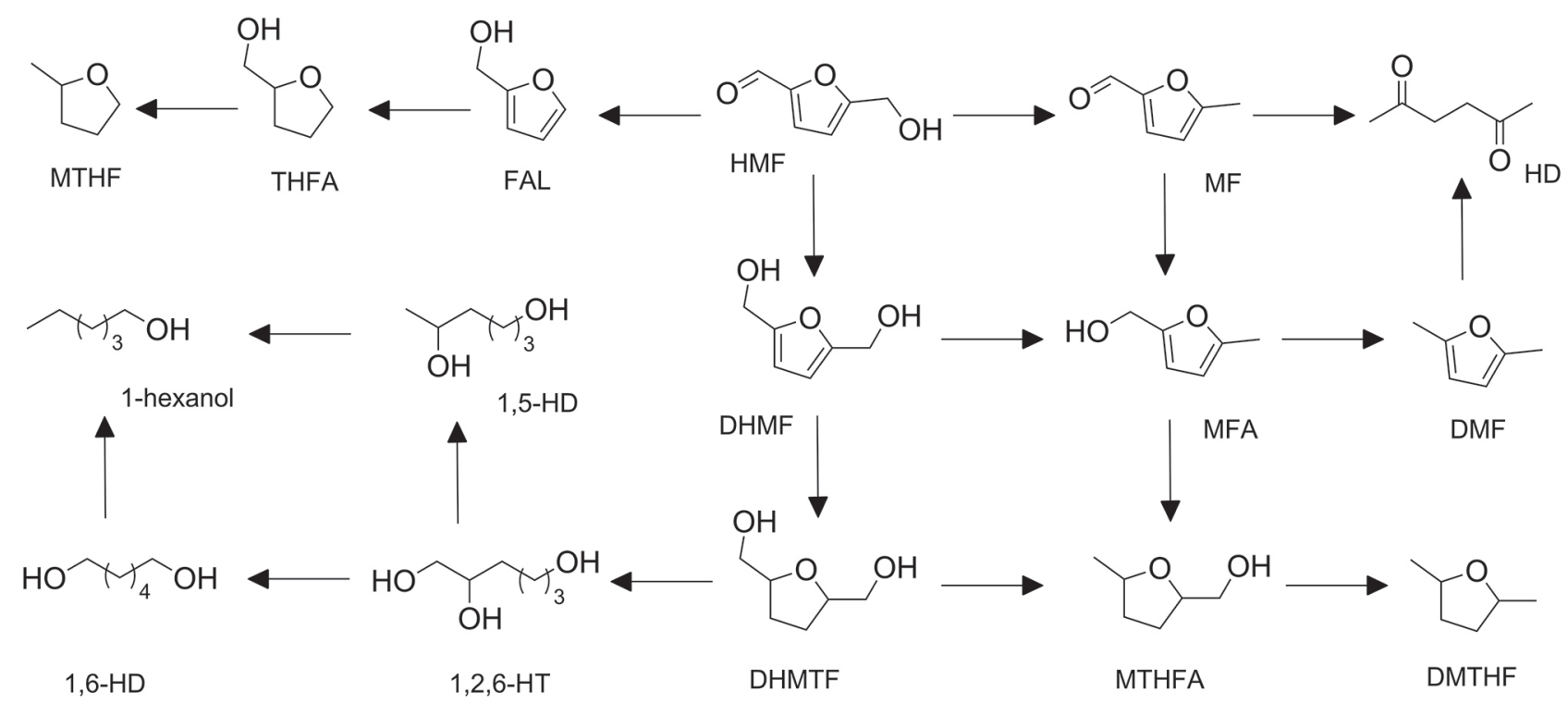

Scheme 1. Possible reaction pathway of 5-HMF hydrogenation and hydrogenolysis.

hydrogenation of $\mathrm{C}=\mathrm{O},{ }^{24}$ but the preferential hydrogenation of the carbonyl groups occurs on gold. ${ }^{14,25,26}$ Van Leeuwen and co-workers ${ }^{9,10}$ reported the cooperative effect of ligands (secondary phosphine oxides, SPO) adsorbed on gold for the selective hydrogenation of $\alpha, \beta$-unsaturated aldehydes to the corresponding allylic alcohols. Density functional theory (DFT) calculations revealed a mechanism based on the heterolytic cleavage of $\mathrm{H}_{2}$ via FLPs at the SPO-gold interface. ${ }^{11}$ Ouyang and Jiang ${ }^{27}$ have also discussed the possibility of a heterolytic dissociation of $\mathrm{H}_{2}$ at the goldbenzalacetone interface. The $\mathrm{Au}^{\delta+---} \mathrm{H}^{\delta-}-\mathrm{H}^{\delta+---} \mathrm{O}^{\delta-}=\mathrm{C}^{\delta+}-\mathrm{R}$ would explain the preferential hydrogenation of $\mathrm{C}=\mathrm{O}$ over $\mathrm{C}=\mathrm{C}$ with gold catalyst.

Considering the interest in the selective hydrogenation of bio-based chemical building blocks containing aldehydes that have many selectivity issues, here we explored their conversion on gold-ligand catalyst systems developed in our group for the hydrogenation of alkynes. We found that gold catalysts can be excellent candidates for the hydrogenation of carbonyl group without opening the furanic ring or reducing $\mathrm{C}-\mathrm{C}$ double bonds.

\section{Experimental}

\section{General information}

Unless otherwise stated, all the reagents used for the support and catalyst preparation were of analytical grade, purchased from Sigma-Aldrich (St. Louis, MO, USA) and used as received. Tetrachloroauric(III) acid was purchased as a $30 \mathrm{wt} . \%$ aqueous solution in dilute $\mathrm{HCl}$. The glass reactor was thoroughly cleaned with aqua regia
$\left(\mathrm{HCl}: \mathrm{HNO}_{3}=3: 1 \mathrm{v} / \mathrm{v}\right)$, rinsed with copious pure water, and then dried in an oven prior to use.

Metal leaching into the supernatant solution was measured by inductively coupled plasma optical emission spectrometry (ICP OES) measurements, performed on a Spectro Arcos ICP OES. Gas chromatography (GC) analyses were carried out with a Shimadzu GC-2010 equipped with an RTx-5 column $(30 \mathrm{~m} \times 0.25 \mathrm{~mm} \times 0.25 \mathrm{~mm})$ and flame ionization detector (FID). Method: initial temperature $=40^{\circ} \mathrm{C}$; final temperature $=200^{\circ} \mathrm{C} ; 17 \mathrm{~min}$; FID and split injector temperatures $=200{ }^{\circ} \mathrm{C}$; internal standard: biphenyl.

\section{Procedure for preparation of $\mathrm{Au} / \mathrm{SiO}_{2}$}

Gold nanoparticles were prepared by the depositionreduction method reported elsewhere. ${ }^{8} \mathrm{~A}$ mass of $1.0 \mathrm{~g}$ of amino-modified silica was added to an aqueous solution of $\mathrm{HAuCl}_{4}\left(20 \mathrm{~mL}, 1.3 \times 10^{-2} \mathrm{~mol} \mathrm{~L}^{-1}\right)$ under continuous stirring. After $30 \mathrm{~min}$, the mixture was centrifugated and the recovered solid was dispersed in $10 \mathrm{~mL}$ of water. Then, under vigorous stirring a freshly-prepared cold aqueous $\mathrm{NaBH}_{4}$ solution ( $\left.10 \mathrm{~mL}, 0.2 \mathrm{~mol} \mathrm{~L}^{-1}\right)$ was added dropwise. After further stirring for $20 \mathrm{~min}$, the solid was recovered by filtration and thoroughly washed twice with hot water $\left(60{ }^{\circ} \mathrm{C}\right)$, acetone and dried in vacuum. The percentage of gold in the fresh catalyst was determined by flame atomic absorption spectroscopy (FAAS) as 1.8 wt.\%.

\section{Procedure for preparation of $\mathrm{Au} @ \mathrm{~N}$-doped carbon / $\mathrm{TiO}_{2}$}

Gold nanoparticles embedded on N-doped carbon 
were prepared by the pyrolysis method reported elsewhere. ${ }^{28}$ Typically, $\mathrm{Au}(\mathrm{OAc})_{3}(19.0 \mathrm{mg}, 0.05 \mathrm{mmol})$ and 1,10-phenanthroline $(18.0 \mathrm{mg}, 0.1 \mathrm{mmol})$ (molar ratio Au:ligand $=1: 2$ ) were stirred in $20 \mathrm{~mL}$ of ethanol for approximately $5 \mathrm{~min}$ at $60{ }^{\circ} \mathrm{C}$. The support was then added, and the mixture was stirred for more $30 \mathrm{~min}$. After, the solvent was removed using a rotary evaporator. The as obtained solid was ground to a fine powder and then pyrolyzed in the desired temperature in an oven at a rate of $20^{\circ} \mathrm{C}$ per minute and held at $400{ }^{\circ} \mathrm{C}$ for $2 \mathrm{~h}$ under $\mathrm{N}_{2}$ atmosphere. The percentage of gold in the fresh catalyst was determined by FAAS as 1.1 wt. $\%$.

\section{General procedure for hydrogenation of aldehydes}

A typical procedure for the hydrogenation of aldehydes is as follows: aldehyde ( $1 \mathrm{mmol})$, Au catalyst, and $2 \mathrm{~mL}$ of solvent were placed in a modified Fischer-Porter $100 \mathrm{~mL}$ glass reactor. The reactor was purged five times with $\mathrm{H}_{2}$, leaving the vessel at the desired pressure. The resulting mixture was vigorously stirred, and the temperature was maintained with an oil bath. After the desired time, the catalyst was removed by centrifugation and the products were analyzed by GC with an internal standard to determine the conversion and selectivity.

\section{Recycling experiment}

For the recycle experiments, after each catalytic experiment under the above typical reaction conditions, the catalyst was recovered from the reaction system by centrifugation. The used catalyst was tested again under identical reaction conditions just by adding to the reactor a new amount of substrate and solvent. The product obtained after centrifugation was analyzed by GC with an internal standard to determine the conversion and selectivity.

\section{Results and Discussion}

At the outset of our studies we chose benzaldehyde 1a (Table 1) as model substrate, which was hydrogenated at 6 bar of $\mathrm{H}_{2}$ pressure and $80{ }^{\circ} \mathrm{C}$ in the presence of gold nanoparticles supported on silica (mean diameter $3.2 \pm 0.6 \mathrm{~nm}$ ). We initially applied the procedure elaborated for the semihydrogenation of alkynes using piperazine as ligand, as reported elsewhere. ${ }^{8}$ Even though the reduction of benzaldehyde has occurred (conversion of 1a $62 \%$ ), the main product detected was the secondary amine formed by coupling of the ligand with the aldehyde. Alternative approaches by adding tertiary amines as ligands were investigated. Among the ligands only L2 and L3 (Table 1, entries 2 and 3) afforded an increase in the catalytic activity, the reaction conversions were 72 and $90 \%$, respectively. Unfortunately, it was not possible to verify the same effect after adding other ligands (Table 1, entries 1 and 4-8), in fact in some cases a decrease in the catalytic activity was noticed, which might occur due to strong adsoption of the ligands to the gold surface, blocking the catalytic active sites. ${ }^{8,29}$ Importantly, a blank experiment without any ligand led to $60 \%$ conversion of $\mathbf{1 a}$, giving us insights to believe that the carbonyl compound might assist gold in the heterolytic $\mathrm{H}_{2}$ dissociation, leading to the formation of

Table 1. Gold-catalyzed reduction of benzaldehyde using $\mathrm{H}_{2}$ :ligand screening

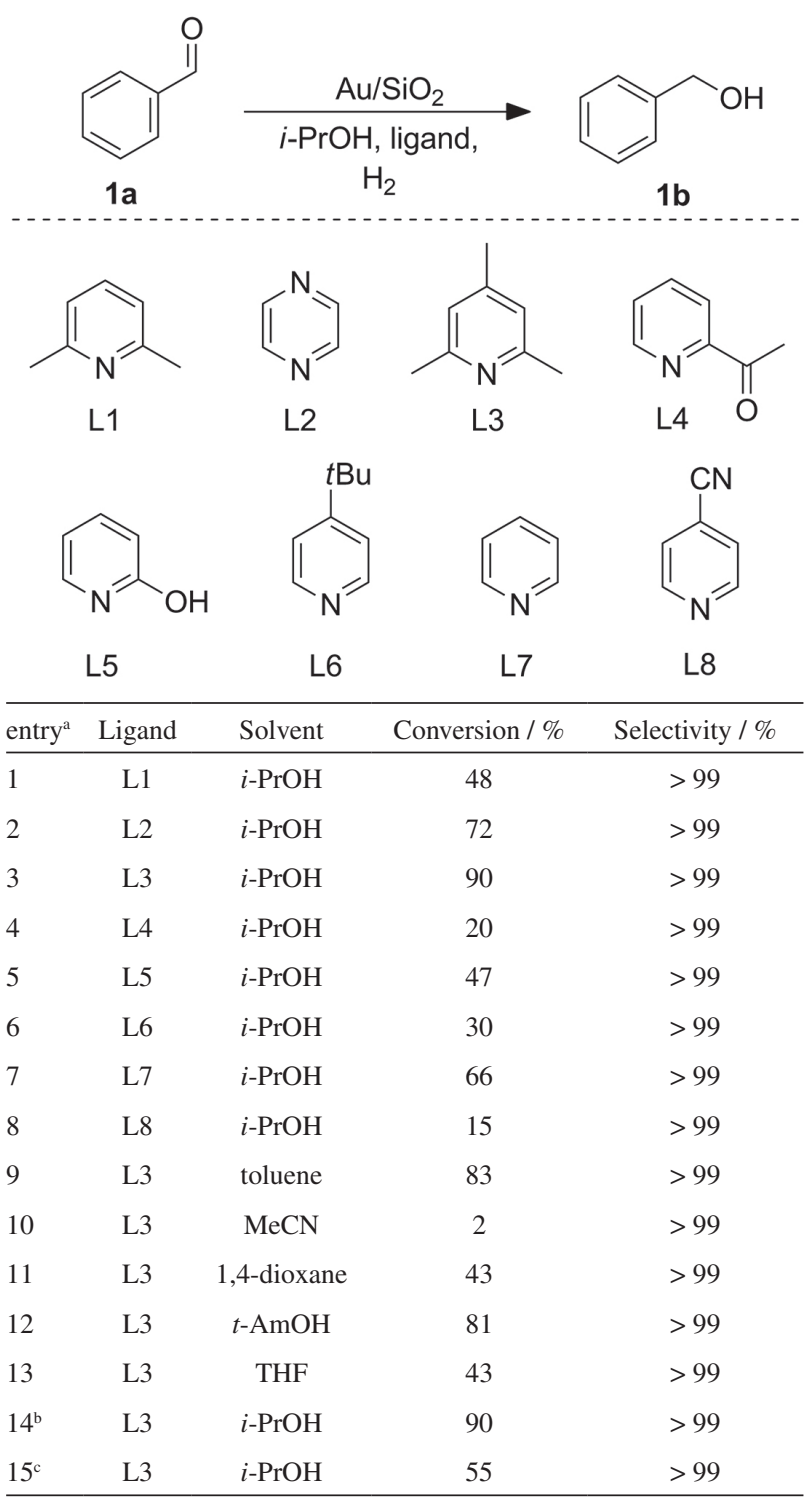

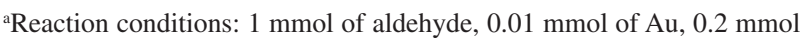
of ligand, $2 \mathrm{~mL}$ of solvent, $100{ }^{\circ} \mathrm{C}, 6$ bar of $\mathrm{H}_{2}, 24 \mathrm{~h}$. Conversion and selectivity were determined by $\mathrm{GC}$ analysis using biphenyl as an internal standard; ${ }^{b} 80^{\circ} \mathrm{C} ;{ }^{\mathrm{c}} 60^{\circ} \mathrm{C}$. THF: tetrahydrofuran. 
a hydride on the metal surface and a proton which bonds to the carbonyl oxygen. ${ }^{11,27}$ Hydrogenation studies were continued, under different reaction conditions, with ligand L3 (2,4,6-trimethylpyridine or collidine) that reached higher conversions. There is no significant effect of the solvent polarity; however, polar aprotic solvents such as acetonitrile (Table 1, entry 10), 1,4-dioxane (Table 1, entry 11) or THF (Table 1, entry 13) showed a significant decrease in the catalytic activity. In fact, polar protic solvents can further facilitate hydrogenation of carbonyl compounds, since the reaction mechanism might occur via dipolar interaction and the $\mathrm{C}=\mathrm{O}$ group may be stabilized by solvent. Moreover the solvent can play a role in assisting to stabilize the intermediates in the reaction and slightly lowering the barrier of $\mathrm{H}_{2}$ cleavage. ${ }^{27}$

The catalytic system obtained using L3 as ligand gave the best catalytic result among the ligands tested and was used for further studies. Notably, no reaction took place in the absence of catalyst or $\mathrm{H}_{2}$. We further examined the effect of the amount of ligand added to the reaction (Figure S1, Supplementary Information (SI) section). Contrary from what was previously observed by our group in the hydrogenation of alkynes, ${ }^{8}$ in this case using only 5 equiv. of ligand (ligand:Au) led to the best catalytic system, with full conversion of $\mathbf{1 a}$ giving $\mathbf{1 b}$ as the only product observed. A slight decrease was noticed with an increase in the amount of added ligand up to 100 equiv. The kinetic profile of the reaction (Figure S2, SI section) reveals that benzaldehyde 1a was converted efficiently into benzyl alcohol $\mathbf{1 b}$ without significant formation of any side products, and the reaction was complete in about $24 \mathrm{~h}$. It is worth to note that the productivity at low conversion (30\% of conversion of 1a) was $582 \mathrm{~mol} \mathrm{~mol}^{-1} \mathrm{~h}^{-1}$. The productivity of the reaction catalyzed by the developed system is higher than previous literature applying gold heterogeneous catalyst using molecular hydrogen or any other reductant (Table S1, SI section).

With optimized reaction conditions in hand, we were keen to demonstrate the substrate scope and functional groups tolerated by the developed catalytic system (Table 2). The reaction appears to be very general, and in most cases the aldehydes were reduced to alcohols in good yields, including some that are of particular interest in the production of perfumes and fragrances. ${ }^{30}$ Cinnamyl alcohol (2h) was obtained in good selectivity (84\%). The hydrogenation of 4-nitrobenzaldehyde provided quantitative conversion, but 4-amino-benzaldehyde was the main product, resulting from the preferential reduction of the nitro group into the amine product. Of particular importance, the developed catalytic system was able to hydrogenate bio-derived furanic aldehydes with excellent yield of the desired alcohols ( $\mathbf{2} \mathbf{i}$ and $\mathbf{2} \mathbf{j}$ ).

In order to support our previous assertion that homogeneous catalytic species are not formed by metal leaching during the reaction, a hot-filtration test was performed (Figure S3a, SI section). For this purpose, a catalysis experiment was run to about $50 \%$ conversion and then stopped. The heterogeneous catalyst $\left(\mathrm{Au} / \mathrm{SiO}_{2}\right)$ was removed by filtration and the reaction mixture was again exposed to reaction conditions. No further conversion was obtained from the supernatant, suggesting that the catalysis was heterogeneous in nature. The recovered solid catalyst

Table 2. Substrate scope of aldehyde hydrogenation catalyzed by $\mathrm{Au} / \mathrm{SiO}_{2}{ }^{\mathrm{a}}$

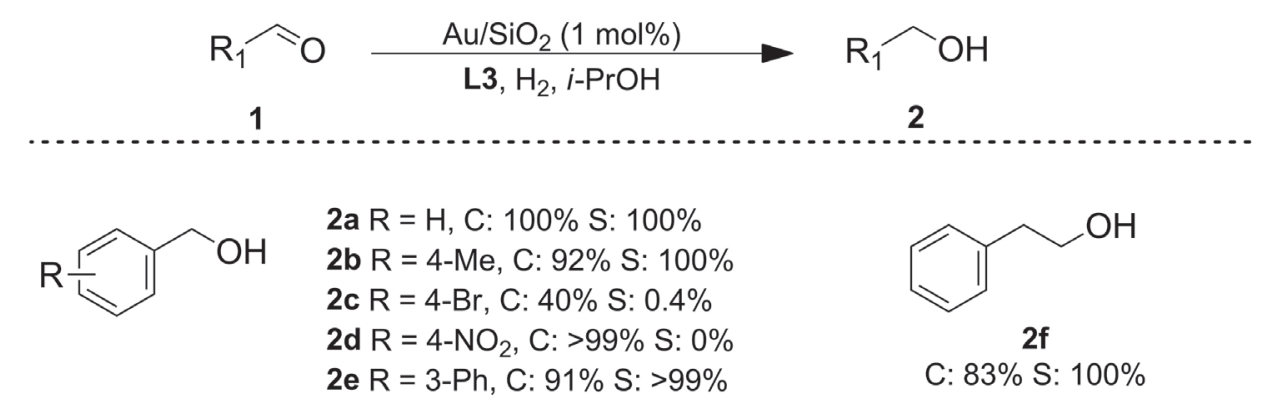

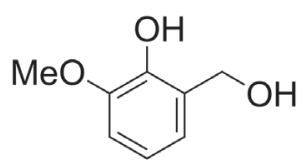

$2 \mathrm{~g}$

C: $100 \%$ S: $86 \%$

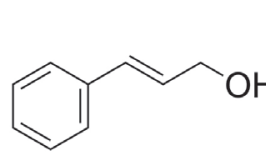

$2 \mathrm{~h}$

C: $65 \%$ S: $84 \%$

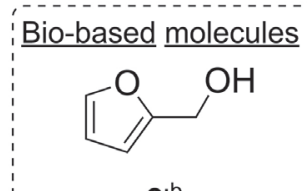

$2 \mathrm{i}^{\mathrm{b}}$

C: $>99 \%$ S: $>99 \%$

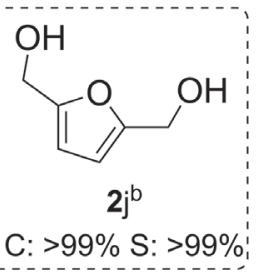

aReaction conditions: $1 \mathrm{mmol}$ of aldehyde, $0.01 \mathrm{mmol}$ of Au, $0.05 \mathrm{mmol}$ of ligand, $2 \mathrm{~mL}$ of $i$-PrOH, $100{ }^{\circ} \mathrm{C}, 6$ bar of $\mathrm{H}_{2}, 24 \mathrm{~h}$. Conversion and selectivity were determined by $\mathrm{GC} ;{ }^{\mathrm{b}} 100{ }^{\circ} \mathrm{C}$. 
showed the same activity and selectivity as the fresh catalyst and no appreciable loss of catalytic activity and selectivity was observed after five cycles (Figure S3b, SI section).

Based on the preferential reduction of nitro groups in the presence of an aldehyde, we tested the possibility of synthesizing secondary amines directly from widely available nitro-compounds and an aldehyde, in one pot reductive amination reaction using our catalytic system. As a test reaction, nitrobenzene and furfural were used for the production of furfurylaniline (Scheme 2). The Au-ligand L3 catalytic system was able to catalyze the reductive amination of $\mathbf{4 a}$ in the presence of $\mathbf{1 i}$ into the desired secondary amine (5a) with good to excellent yield (up to 99\% yield) and selectivity. Furfurylamines are important intermediates in the synthesis of pharmaceuticals such as antiseptic agents, antihypertensives, and diuretics (e.g., furosemide). ${ }^{3}$

In spite of the good results regarding the selectivity for aldehyde reduction using amine-ligands and Au NPs, the presence of a ligand in the reaction media hinders the development of a continuous flow process, in line with the best green chemical principles. Therefore, a fully heterogeneous gold catalyst (Au@N-doped-carbon / $\mathrm{TiO}_{2}$ ) developed by our group was tested. ${ }^{28}$ Initial experiments were aimed at evaluating the feasibility of Au-catalyzed aldehyde reduction using $\mathrm{H}_{2}$ as hydride source under "amine ligand-free" conditions. As a preliminary test, the reductive conversion of benzaldehyde 1a was carried out, with the $\mathrm{Au} @ \mathrm{~N}$-doped-carbon / $\mathrm{TiO}_{2}$ catalyst $(2 \mathrm{~mol} \%)$ in $i$-PrOH at $100{ }^{\circ} \mathrm{C}$ (Table 3). The obtained results show that the corresponding alcohol, benzyl alcohol 2a, was selectively obtained without any side product (Table 3 , entry 1 ). This result underscores the already high performance of our nitrogen-doped carbon gold catalyst, and thus expands its application. As expected, the heterogenized catalyst without pyrolysis, a catalyst version without the carbon doping structure and the support with the nitrogen-carbon doped structure did not show any activity (Table 3, entry 2-4). Notably, at lower hydrogen pressure $\left(10 \mathrm{bar}_{2}\right)$ the reaction was retarded (Table 3 , entry 5).

Next, a variety of bio-based aldehydes were hydrogenated to their corresponding alcohols with high efficiency (Table 4). For the hydrogenation of the cinnamaldehyde $1 \mathbf{h}$ into cinnamyl alcohol $2 \mathrm{~h}$, the catalyst provided high activity and moderated selectivity. The heterocyclic furanic compounds, furfural and 5-HMF, can also be efficiently hydrogenated to form furfuryl alcohol $2 \mathbf{i}$ and DHMF $\mathbf{2 j}$. With a further increase in $\mathrm{H}_{2}$ pressure,

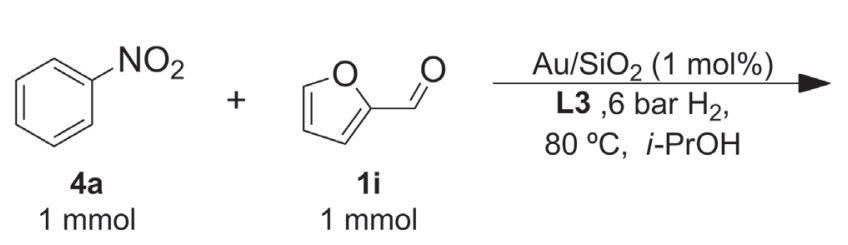

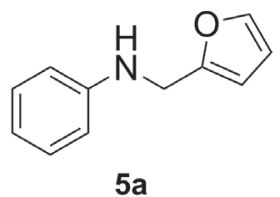

Conversion 4a: $100 \%$ Selectivity 5a: $100 \%$

Scheme 2. Au-catalyzed reductive amination of nitrobenzene and furfural.

Table 3. Hydrogenation of benzaldehyde catalyzed by Au@N-doped carbon/ $/ \mathrm{TiO}_{2}$

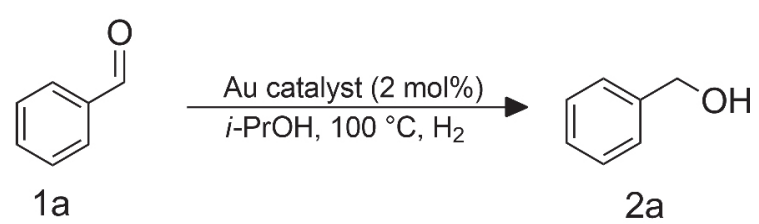

\begin{tabular}{lcccc}
\hline entry & Catalyst & $\mathrm{H}_{2}$ pressure / bar & Conversion of 1a / \% & Selectivity to 2a / \% \\
\hline 1 & $\mathrm{Au} @ \mathrm{~N}$-doped carbon / $\mathrm{TiO}_{2}$ & 20 & 100 & $>99$ \\
$2^{\mathrm{b}}$ & $\mathrm{Au}-\mathrm{phen} / \mathrm{TiO}_{2}$ & 20 & 0 & - \\
3 & $\mathrm{Au} / \mathrm{TiO}_{2}$ & 20 & 0 & - \\
$4^{\mathrm{c}}$ & $\mathrm{N}$-doped carbon / $\mathrm{TiO}_{2}$ & 20 & 0 & $>$ \\
5 & $\mathrm{Au} @ \mathrm{~N}$-doped carbon / $\mathrm{TiO}_{2}$ & 10 & 32 & $>99$ \\
\hline
\end{tabular}

aReaction conditions: $0.1 \mathrm{mmol}$ of $\mathbf{1 a}, 2 \mathrm{~mol} \%$ of Au catalyst, $2 \mathrm{~mL}$ of $i$-PrOH, $100{ }^{\circ} \mathrm{C}, \mathrm{H}_{2}, 20 \mathrm{~h}$; conversion and selectivity were determined by $\mathrm{GC}$ using internal standard technique; ${ }^{\mathrm{b}}$ catalyst precursor not pyrolyzed (phen $=1,10$-phenanthroline); ${ }^{\mathrm{c}}$ prepared by pyrolysis of $\mathrm{phen} / \mathrm{TiO}_{2}$ (without gold). 
$\mathrm{Au} @ \mathrm{~N}$-doped-carbon/ $/ \mathrm{TiO}_{2}$ showed high chemoselectivity for the hydrogenation of citral giving nerol and geraniol $\mathbf{2 k}$. Furthermore, vanillin alcohol $\mathbf{2 l}$ was obtained as the only product from hydrogenation of vanillin; no product from hydrodeoxygenation process was detected.

In terms of the stability (Figure 1), a slight decrease in the conversion was observed after the first and second cycles. However, after the second run the catalytic performance remained constant. In addition, the selectivity was completely retained at a value of $>99 \%$ throughout the 5 recycling experiments. In comparison with the Au-amine ligand and other few reported heterogeneous gold-based catalyst applied in the hydrogenation of aldehydes (Table S1, SI section), these results represent a step further in the development of more efficient yet substantially safer means for target-specific reduction reactions. Moreover, the catalytic activity of the developed systems is comparable or superior to previous studies using benchmark Au heterogeneous catalysts, with potential to be studied under flow conditions.

Regarding the reaction mechanism, the fact that the blank reaction without the presence of an added ligand showed around $60 \%$ conversion suggests that the aldehyde, when adsorbing on the gold surface, might assist the heterolytic $\mathrm{H}_{2}$ dissociation, leading to the formation of a hydride on the metal surface and a proton which bonds to the carbonyl oxygen. Ouyang and Jiang ${ }^{27}$ and Lopez et al., ${ }^{11}$ using DFT calculations, have shown that the heterolytic cleavage of $\mathrm{H}_{2}$ can be promoted by the carbonyl compound approaching gold surface, followed by the facile transfer of hydride from gold surface to the partially hydrogenated

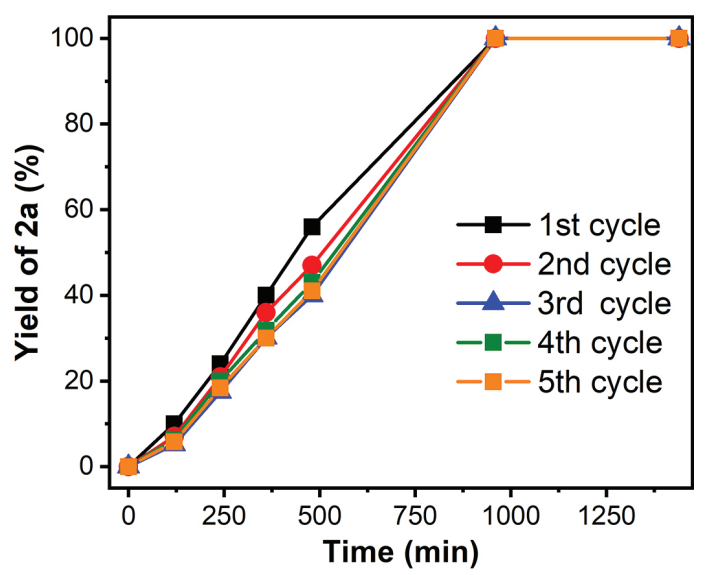

Figure 1. Reuse of $\mathrm{Au} @ \mathrm{~N}$-doped carbon / $\mathrm{TiO}_{2}$ for hydrogenation of benzaldehyde $\mathbf{1 a}$.

substrate. In agreement with our experimental results, DFT calculations also show that the energy barriers for the heterolytic $\mathrm{H}_{2}$ dissociation decrease by adding an auxiliary ligand (calculations are shown for secondary phosphine oxides). ${ }^{11}$ Here, the catalytic activity increased after adding collidine (L3), reaching $100 \%$ conversion of benzaldehyde in the same period of time and under milder temperature conditions than the reaction without any ligand. It is reasonable to suggest that the heterolytic cleavage of $\mathrm{H}_{2}$ occurs at the Au-collidine interface and similarly at the Au@N-doped-carbon interface (in the heterogeneous catalyst), forming hydrides on the gold surface. This step was experimetally and theoretically demonstrated before for the alkyne-to-alkene hydrogenationwith Au@Ndoped carbon catalyst. ${ }^{28}$ It is reasonable to suggest that the aldehyde hydrogenation into the desired alcohol takes

Table 4. Hydrogenation of aldehydes catalyzed by Au@ N-doped carbon / $\mathrm{TiO}_{2}{ }^{\mathrm{a}}$

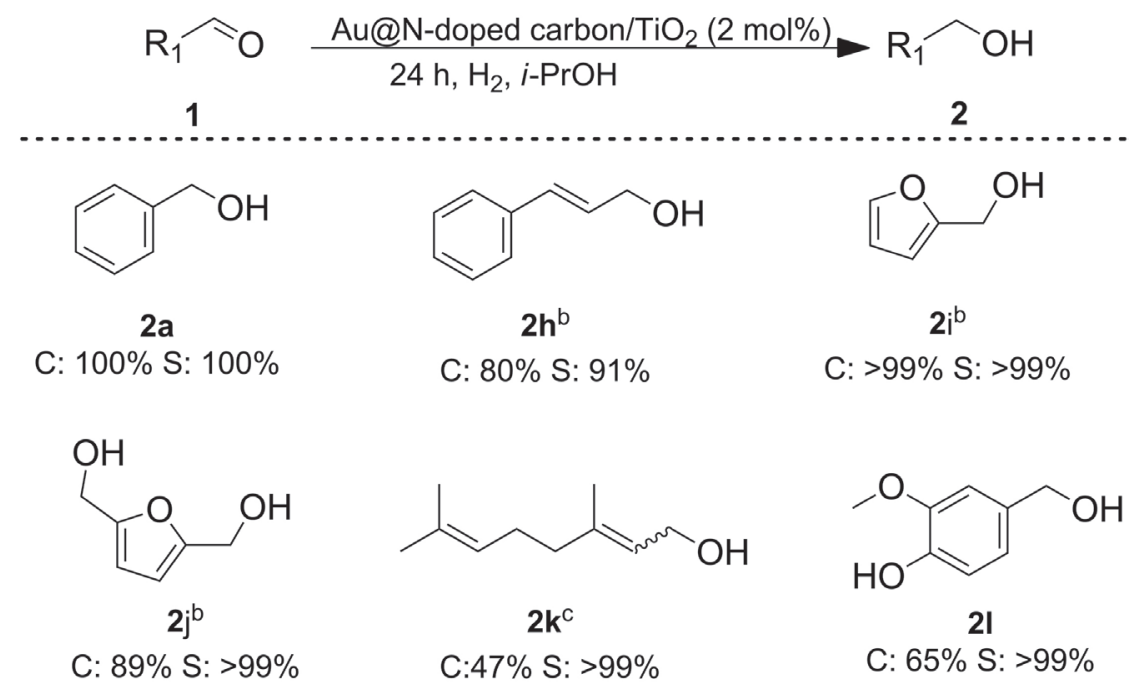

aReaction conditions: $1 \mathrm{mmol}$ of aldehyde, $2 \mathrm{~mol} \%$ of Au, $2 \mathrm{~mL}$ of $i$-PrOH, $100{ }^{\circ} \mathrm{C}, 20$ bar of $\mathrm{H}_{2}, 24 \mathrm{~h}$. Conversion and selectivity were determined by GC; 30 bar of $\mathrm{H}_{2}$; ${ }^{4} 40$ bar of $\mathrm{H}_{2}$. 


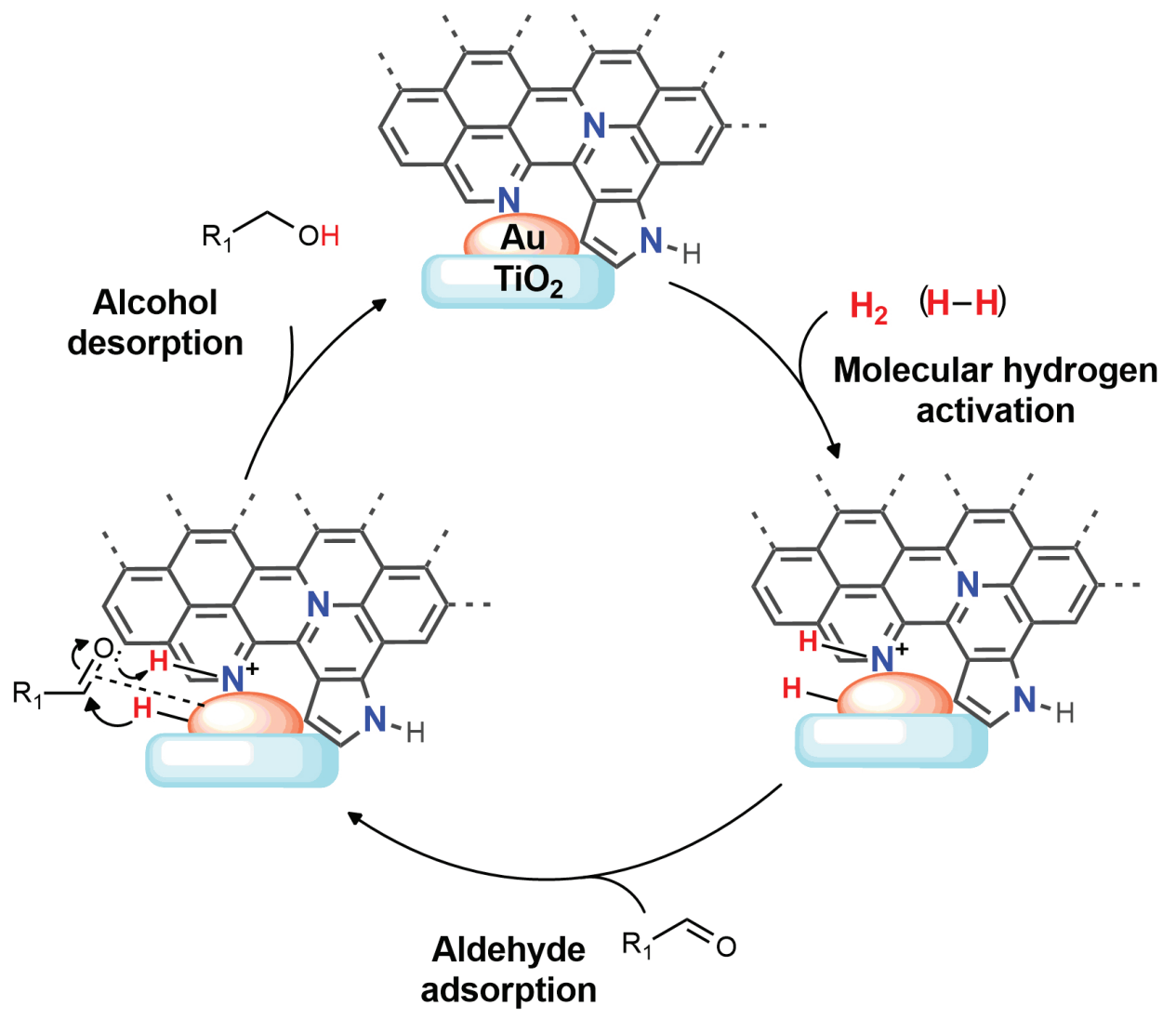

Scheme 3. Proposed mechanism for gold-catalyzed hydrogenation of aldehydes.

place in a concerted step, resulting in the addition of the $\mathrm{H}^{+} / \mathrm{H}^{-}$pair to the $\mathrm{C}=\mathrm{O}$ bond (Scheme 3 ).

\section{Conclusions}

In summary, Au NPs are very selective catalysts for the reduction of aldehydes to the corresponding alcohols using molecular hydrogen as the reductant and become more active in the presence of Lewis bases. The introduction of either a tertiary amine ligand or nitrogen-doped carbon structure greatly improved the catalytic activity of the Au NPs, possibly due to a cooperative effect between gold surface and ligand or N-carbon structure, which can be rationalized as a frustrated Lewis pairs interface that leads to the heterolytic activation of $\mathrm{H}_{2}$, boosting the activity of $\mathrm{Au}$ NPs in reduction reactions. The fully heterogeneous gold catalyst based of N-doped carbon concept (Au@N-doped carbon $/ \mathrm{TiO}_{2}$ ) was able to perform these reactions in successive recycles, allowing the future development of a continuous flow process for biomass valorization.

\section{Supplementary Information}

Supplementary information (additional catalytic tests and a comparative table with similar catalysts from the literature) is available free of charge at http://jbcs.sbq.org.br as PDF file.

\section{Acknowledgments}

The authors are grateful to INCT-Catálise and the Brazilian government agencies FAPESP (2016/16738-7), CNPq, and CAPES for financial support. We also thank Prof Núria López for helpful discussions.

\section{References}

1. van Putten, R. J.; van der Waal, J. C.; de Jong, E.; Rasrendra, C. B.; Heeres, H. J.; de Vries, J. G.; Chem. Rev. 2013, 113, 1499.

2. Kong, X.; Zhu, Y.; Fang, Z.; Kozinski, J. A.; Butler, I. S.; Xu, L.; Song, H.; Wei, X.; Green Chem. 2018, 20, 3657.

3. Dunbabin, A.; Subrizi, F.; Ward, J. M.; Sheppard, T. D.; Hailes, H. C.; Green Chem. 2017, 19, 397.

4. Gupta, K.; Rai, R. K.; Singh, S. K.; ChemCatChem 2018, 10, 2326.

5. Lima, S.; Chadwick, D.; Hellgardt, K.; RSCAdv. 2017, 7, 31401.

6. Bottari, G.; Kumalaputri, A. J.; Krawczyk, K. K.; Feringa, B. L.; Heeres, H. J.; Barta, K.; ChemSusChem 2015, 8, 1323.

7. Chen, J.; Ge, Y.; Guo, Y.; Chen, J.; J. Energy Chem. 2018, 27, 283. 
8. Fiorio, J. L.; López, N.; Rossi, L. M.; ACS Catal. 2017, 7, 2973.

9. Cano, I.; Chapman, A. M.; Urakawa, A.; van Leeuwen, P. W. N. M.; J. Am. Chem. Soc. 2014, 136, 2520.

10. Cano, I.; Huertos, M. A.; Chapman, A. M.; Buntkowsky, G.; Gutmann, T.; Groszewicz, P. B.; van Leeuwen, P. W. N. M.; J. Am. Chem. Soc. 2015, 137, 7718.

11. Almora-Barrios, N.; Cano, I.; van Leeuwen, P. W. N. M.; López, N.; ACS Catal. 2017, 7, 3949.

12. Shibata, M.; Kawata, N.; Masumoto, T.; Kimura, H.; J. Chem. Soc., Chem. Commun. 1988, 154.

13. Zhu, Y.; Qian, H.; Drake, B. A.; Jin, R.; Angew. Chem., Int. Ed. 2010, 49, 1295.

14. Milone, C.; Tropeano, M. L.; Gulino, G.; Neri, G.; Ingoglia, R.; Galvagno, S.; Chem. Commun. 2002, 104, 868.

15. Shi, H.; Xu, N.; Zhao, D.; Xu, B.-Q.; Catal. Commun. 2008, 9 , 1949.

16. Li, G.; Abroshan, H.; Chen, Y.; Jin, R.; Kim, H. J.; J. Am. Chem. Soc. 2015, 137, 14295.

17. Yan, M.; Jin, T.; Ishikawa, Y.; Minato, T.; Fujita, T.; Chen, L.-Y.; Bao, M.; Asao, N.; Chen, M.-W.; Yamamoto, Y.; J. Am. Chem. Soc. 2012, 134, 17536.

18. Li, G.; Jin, R.; J. Am. Chem. Soc. 2014, 136, 11347.

19. Mitsudome, T.; Yamamoto, M.; Maeno, Z.; Mizugaki, T.; Jitsukawa, K.; Kaneda, K.; J. Am. Chem. Soc. 2015, 137, 13452.
20. Shimizu, K.; Miyamoto, Y.; Kawasaki, T.; Tanji, T.; Tai, Y.; Satsuma, A.; J. Phys. Chem. C 2009, 113, 17803.

21. Ren, D.; He, L.; Yu, L.; Ding, R.-S.; Liu, Y.-M.; Cao, Y.; He, H.-Y.; Fan, K.-N.; J. Am. Chem. Soc. 2012, 134, 17592.

22. Noujima, A.; Mitsudome, T.; Mizugaki, T.; Jitsukawa, K.; Kaneda, K.; Chem. Commun. 2012, 48, 6723.

23. Noujima, A.; Mitsudome, T.; Mizugaki, T.; Jitsukawa, K.; Kaneda, K.; Angew. Chem., Int. Ed. 2011, 50, 2986.

24. Vetere, V.; Merlo, A. B.; Ruggera, J. F.; Casella, M. L.; J. Braz. Chem. Soc. 2010, $21,914$.

25. You, K.; Chang, C.; Liaw, B.; Huang, C.; Chen, Y.; Appl. Catal., A 2009, 361, 65.

26. Claus, P.; Appl. Catal., A 2005, 291, 222.

27. Ouyang, R.; Jiang, D.; ACS Catal. 2015, 5, 6624.

28. Fiorio, J. L.; Gonçalves, R. V.; Teixeira-Neto, E.; Ortuño, M. A.; López, N.; Rossi, L. M.; ACS Catal. 2018, 8, 3516.

29. Domingo, M.; Shahrokhi, M.; Remediakis, I. N.; Lopez, N.; Top. Catal. 2018, 61, 412.

30. Zhao, M.; Yuan, K.; Wang, Y.; Li, G.; Guo, J.; Gu, L.; Hu, W.; Zhao, H.; Tang, Z.; Nature 2016, 539, 76.

Submitted: February 12, 2019

Published online: May 21, 2019 